\title{
Charge dependent particle correlations motivated by chiral mag- netic effect and chiral vortical effect
}

\author{
Jie Zhao ${ }^{1, a}$ (for the STAR collaboration) \\ ${ }^{1}$ Department of Physics and Astronomy, Purdue University, West Lafayette, IN 47907, USA
}

\begin{abstract}
In the early stage of relativistic heavy-ion collisions, a strong magnetic field can be generated. The interaction between the strong magnetic field and the hot medium would provide a way to study the nontrivial QCD topological structures, such as the chiral magnetic effect (CME) and chiral vortical effect (CVE). The three particle azimuthal correlator has been proposed to measure the separation of electric/baryonic charges with respect to the reaction plane due to the chiral effects. We report the STAR measurements of three particle azimuthal correlations of charged particles and identified particles to search for possible signatures of the CME and CVE in relativistic heavy-ion collisions of various energies and systems.
\end{abstract}

\section{Introduction}

Metastable domains of topological charges can form in a quark gluon plasma with approximate chiral symmetry restoration [1-5]. These topological charges can change the chirality of quarks in those local domains. This can lead to observable charge separation along the direction of the strong magnetic field produced in the early stage of relativistic heavy-ion collisions $[6,7]$. This is called the chiral magnetic effect (CME). A similar effect, called the chiral vortial effect (CVE), in which the vorticity of the collision system replaces the magnetic field, can lead to baryonic charge separation [8]. Measurements of electric/baryonic charge separations from the CME and CVE can therefore provide a means to studying the nontrivial QCD topological structures. An observation of the CME/CVE-induced charge separation would confirm a fundamental property of QCD and is therefore of paramount importance.

Experimentally, the three particle correlator (Eq. 1) has been proposed [7] to measure the possible signal of CME and/or CVE.

$$
\gamma \equiv\left\langle\cos \left(\phi_{\alpha}+\phi_{\beta}-2 \Psi_{\mathrm{RP}}\right)\right\rangle=\left\langle\cos \left(\phi_{\alpha}+\phi_{\beta}-2 \phi_{c}\right)\right\rangle / v_{2}
$$

Previous experimental results from STAR [9-12], PHENIX [13], and ALICE [14] are consistent with theoretical expectation of CME. However, those results suffer from possible physics background [7, 15]). In this paper, we will discuss new approaches to suppress the flow background, and also report results in small system $d+A u$ collisions at STAR.

\footnotetext{
ae-mail: zhao656@purdue.edu
} 


\section{Experiment setup}

The data used in this analysis were collected by the STAR detector [16] at Brookhaven National Laboratory from year 2003, 2004, 2007, 2010 and 2011. The main subsystems of the STAR detector used for this analysis are the Time Projection Chamber (TPC) [17] and the Time-Of-Flight detector (TOF) [18] with $2 \pi$ azimuthal coverage at mid-rapidity, and three trigger subsystems, the Central Trigger Barrel (CTB), the Vertex Position Detectors (VPDs) and the Zero Degree Calorimeters (ZDCs).

Charged particle identification is performed by ionization energy loss $(d E / d x)$ measured by the TPC and/or particle velocity measured by TOF. The correlations are reported for particles with pseudorapidity $|\eta|<1$ and transverse momentum $p_{T}>0.15 \mathrm{GeV} / \mathrm{c}$. We use the TPC to reconstruct the 2nd order harmonic event plane. The 1st order harmonic event plane is reconstructed by the two Zero Degree Calorimeter Shower Maximum Detectors (ZDC-SMDs) [19], which are sensitive to the directed flow of neutrons in the beam rapidity region.

\section{Charge dependent correlator measurements}

The three particle correlator (Eq. 1) has been proposed [7] to measure charge separation signal: Charge separation along the magnetic field $(\vec{B})$, perpendicular to $\Psi_{\mathrm{RP}}$ on average, would yield positive and negative values of $\gamma$ for particle pairs of opposite-sign (OS) and same-sign (SS) charges, respectively. A positive $\Delta \gamma \equiv \gamma_{O S}-\gamma_{S S}$ is therefore referred to as the charge separation signal.

Figure 1 presents the OS and SS correlators as function of centrality for Au+Au collisions at $\sqrt{s_{N N}}$ = 7.7-200 GeV measured by STAR [12]. With decreasing beam energy, both OS and SS tend to rise in peripheral collisions. Similar results at $200 \mathrm{GeV} \mathrm{Au+Au}$ from PHENIX [13] and 2.76 TeV Pb+Pb from ALICE [14] were observed. This feature seems to be charge independent and can be explained by momentum conservation and elliptic flow [11]. In most cases, the difference between OS and SS is still present. The difference at low collision energies are consistent with zero within large error bars.

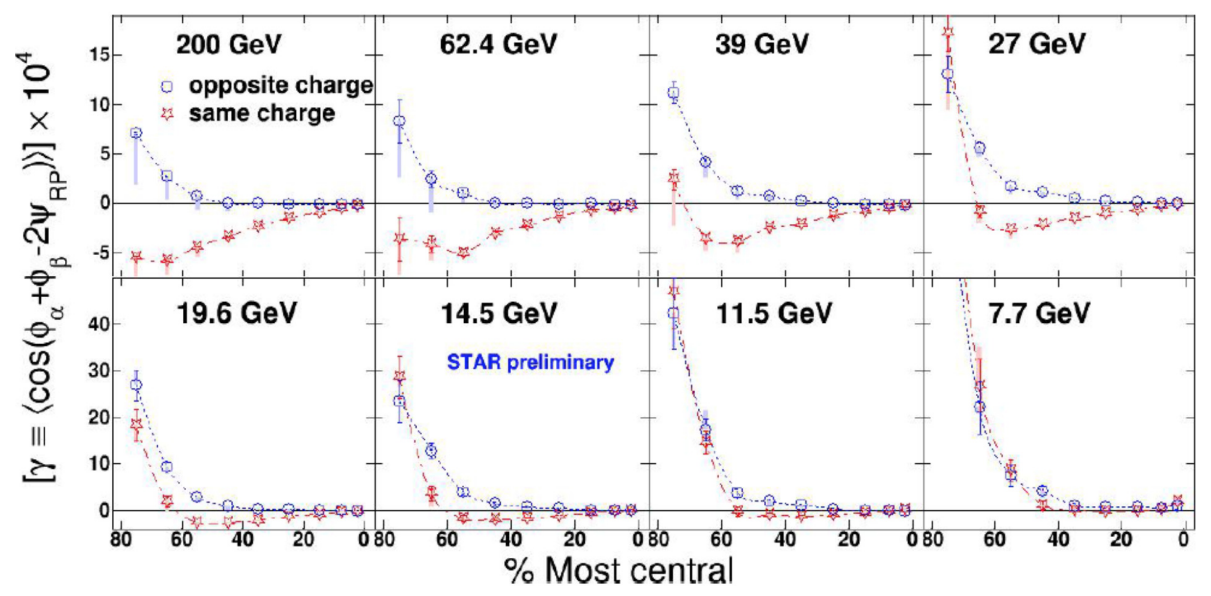

Figure 1. (Color online) Three particle correlator $\gamma$ within $|\eta|<1$ as a function of centrality for Au+Au collisions at 7.7-200 GeV. Note the different vertical scales. The filled bars (starting from the central values) represent the systematic uncertainty [12].

A hierarchal structure of correlation strength is expected from the combination of CME and CVE: p-p $(\mathrm{CME}$ and $\mathrm{CVE})>\mathrm{p}-\Lambda(\mathrm{CVE})>\mathrm{p}-\pi(\mathrm{CME})$. A proton is a charged baryon, so the p-p correlation 
is expected to contain both the electric charge and baryonic charge separation signals; $\mathrm{A} \Lambda$ is a neutral baryon, so the $\mathrm{p}-\Lambda$ is expected to contain only the baryonic charge separation signal; $\mathrm{A} \pi$ is a charged meson, so the $\mathrm{p}-\pi$ is expected to contain only the electric charge separation signal. In order to gain insight on this physics, the identified-particle correlations are used to explore a possible hierarchal structure in the particle dependent correlations [20]. Fig. 2 shows the measured correlation of p-p, $\mathrm{p}-\Lambda$ and $\mathrm{p}-\pi$ for $\mathrm{Au}+\mathrm{Au}$ at $\sqrt{s_{N N}}=200 \mathrm{GeV}$ collisions from STAR. The results are compared to h-h correlation. The measured correlators may be contaminated by physics background (discussed below), and thus can not yet be directly compared to theoretical expectations regarding possible hierarchal structure.

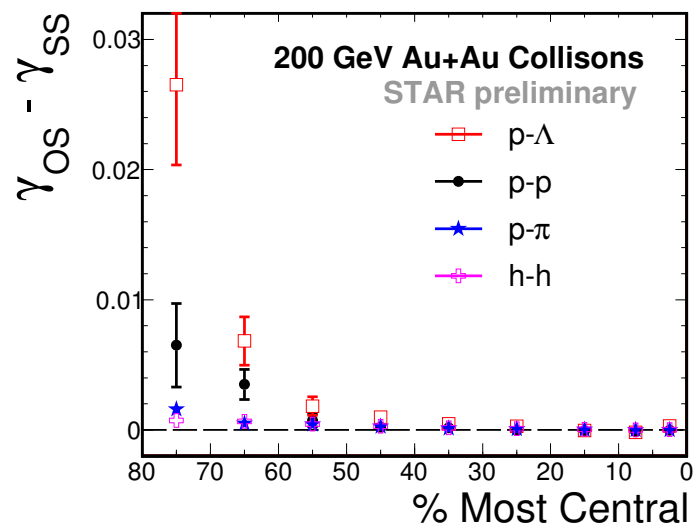

Figure 2. (Color online) Comparison of $\Delta \gamma \equiv \gamma_{O S}-\gamma_{S S}$ between $\mathrm{p}-\Lambda$, $\mathrm{p}-\mathrm{p}, \mathrm{p}-\pi$, h-h as a function of centrality for Au+Au collisions at $\sqrt{s_{N N}}=200 \mathrm{GeV}$ within $|\eta|<1$.

\section{Charge dependent signal with flow background suppression}

While the $\Delta \gamma$ variable gives a positive signal for charge separation along the magnetic field, it also yields a positive signal for charge conservation along the reaction plane direction, for example, a positive-negative charge pair from a resonance decay. Since resonances (or generally, clusters including jets, dijets, etc.) exhibit elliptic anisotropy, there will be an overall positive $\Delta \gamma$. Those "flowing clusters" can thus mimic a CME/CVE signal [7, 10, 15]. In the case of resonance decay, the three particle correlator can be expressed as follows:

$$
\left\langle\cos \left(\phi_{\alpha}+\phi_{\beta}-2 \Psi_{\mathrm{RP}}\right)\right\rangle=\left\langle\cos \left(\Delta \phi+2 \phi_{\text {res. }}-2 \Psi_{\mathrm{RP}}\right)\right\rangle \approx\langle\cos (\Delta \phi)\rangle \cdot v_{2, \text { res }}
$$

where $\alpha$ and $\beta$ are the decay products of resonance (res. $\rightarrow \alpha+\beta), \Delta \phi \equiv \phi_{\alpha}+\phi_{\beta}-2 \phi_{\text {res. }}$, and $v_{2, \text { res }}=\left\langle\cos 2\left(\phi_{\text {res. }}-\Psi_{\mathrm{RP}}\right)\right\rangle$.

To suppress the flow background, STAR has exploited an event-by-event method and extracted the charge dependent signal at the observed event-by-event final-state particle anisotropy $v_{2, \text { ebye }}^{\text {obs }}=0$ [21]. Since this does not necessarily guarantee zero anisotropy of the background sources (such as the resonances), residual physics background may remain [22].

In our analysis, we divide a full TPC event into two sub-events $(-1<\eta<-0$ and $0<\eta<$ 1 ), and calculate the three particle correlator and $v_{2 \text {,ebye }}^{\text {obs }}$ by using the particles from one side of the 
TPC with respect to the event plane reconstructed from the other side of the TPC. Besides using the event-by-event method developed in [21] to extract signal at $v_{2 \text {,ebye }}^{\text {obs }}=0$, we also use the mixed-event method to suppress background. In the mixed-event method, we calculate the correlator and $v_{2 \text {,ebye }}^{\text {obs }}$ with respect to the event plane taken from another event (i.e. we replace the $\Psi_{2}\left(\Psi_{\mathrm{RP}}\right)$ in Eq. 2 with the event plane from another event). We subtract the mixed-event signal from the real-event signal at the same $v_{2 \text {,ebye }}^{\text {obs }}$. We then average the mixed-event subtracted signal from all real events, according to the probability distribution of real events in $v_{2 \text {,ebye }}^{\text {obs }}$. In other words, the subtracted mixed events are effectively weighted by the $v_{2 \text {,ebye }}^{\text {obs }}$ distribution of real events. Although the signal simply averaged in mixed-events is zero, the signal averaged according to the $v_{2 \text {,ebye }}^{\text {obs }}$ probability distribution of the real events (not symmetric about $v_{2 \text {,ebye }}^{\text {obs }}=0$ ) is no longer vanishing.
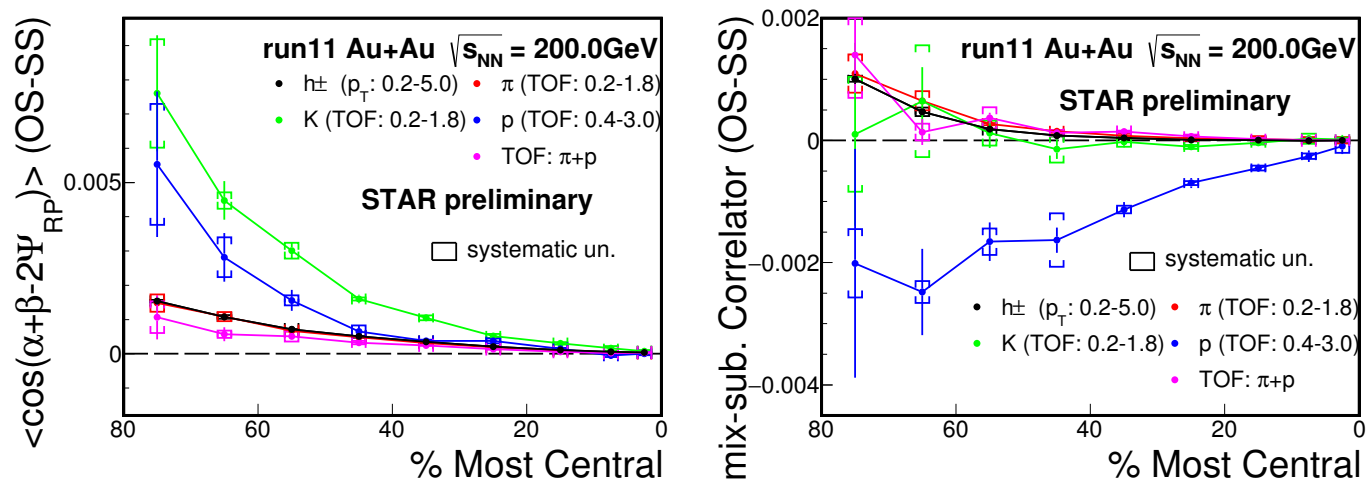

Figure 3. (Color online) Comparison of $\Delta \gamma$ (Left) and mixed-event subtracted $\Delta \gamma$ (Right) for charged particles, and identified particles $(\pi, \mathrm{K}, \mathrm{p})$ as a function of centrality for Au+Au collisions at $\sqrt{s_{N N}}=200 \mathrm{GeV}$. The correlators and $v_{2, \text { ebve }}^{\text {obs }}$ are from one half of the TPC in $\eta$ and the event plane is from the other half of the TPC. The brackets represent the systematic uncertainty.

Figure 3 left panel shows the centrality dependence of $\Delta \gamma$ for charged particles, and identified particles $(\pi, K, p)$ from $\mathrm{Au}+\mathrm{Au}$ collisions at $\sqrt{s_{N N}}=200 \mathrm{GeV}$. Fig. 3 right panel shows the results with the mixed-event background subtraction, which is used to suppress the $v_{2}$ related background. With flow background suppression, the signals are reduced. For charged hardon and charged $\pi$ the signals are reduced by $\sim 50 \%$, while for proton the signal becomes negative.

Figure 4 shows the collision energy dependence of the mixed-event subtracted $\Delta \gamma$ for different centralities in $\mathrm{Au}+\mathrm{Au}$ collisions at $\sqrt{s_{N N}}$ form 7.7 to $200 \mathrm{GeV}$. In central collisions, charge separation signals are consistent with zero. In non-central collisions, the signals are finite, and the energy dependence is weak.

\section{Charge dependent signal in small system}

Recent results from CMS show that the correlator signal from $\mathrm{p}+\mathrm{Pb}$ is comparable to the signal from $\mathrm{Pb}+\mathrm{Pb}$ collisions at similar multiplicities [23]. We have carried out a similar control experiment using $\mathrm{d}+\mathrm{Au}$ collisions. Fig. 5 shows $\Delta \gamma$ in $\mathrm{d}+\mathrm{Au}$, compared to $\mathrm{Au}+\mathrm{Au}$ collisions at $\sqrt{s_{N N}}=200 \mathrm{GeV}$. The data are scaled by the geometric mean of the positive and negative particle multiplicities. We observe sizeable charge dependent signal in small system d+Au collisions, also comparable to 200 $\mathrm{GeV} \mathrm{Au+Au} \mathrm{collisions} \mathrm{at} \mathrm{similar} \mathrm{multiplicities.}$ 


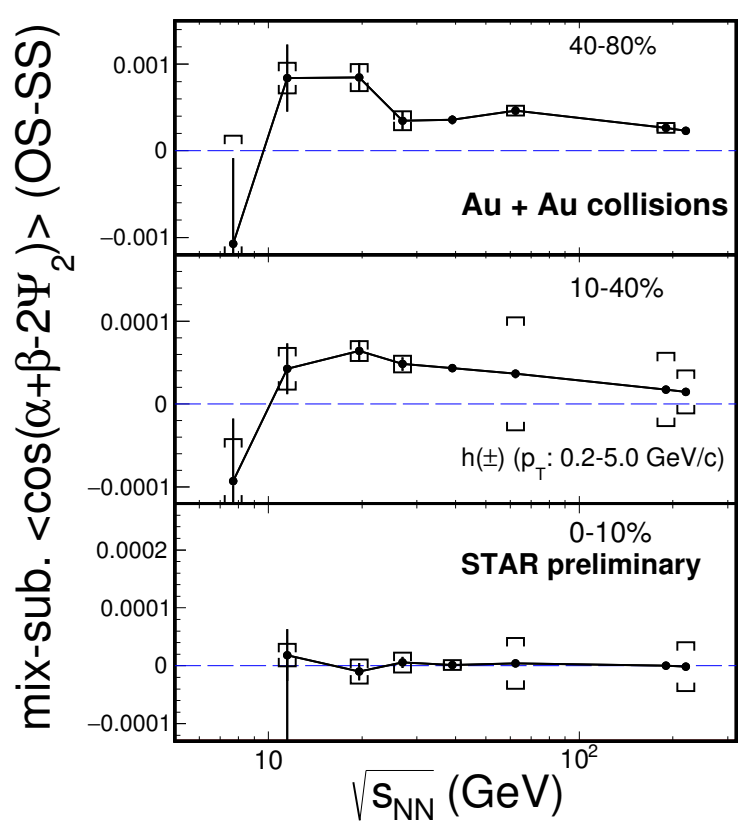

Figure 4. Collision energy dependence of the mixed-event subtracted $\Delta \gamma$ for different centralities in $\mathrm{Au}+\mathrm{Au}$ collisions at $\sqrt{s_{N N}}=200 \mathrm{GeV}$ (Particles contributing to the correlators and $v_{2, \text { ebye }}^{\text {obs }}$ are from one half of the TPC in $\eta$, and the event plane is from the other half of the TPC).

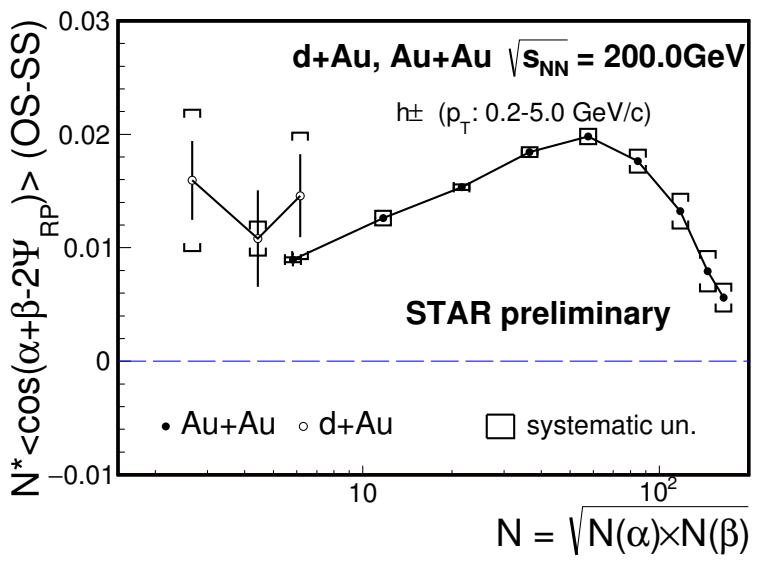

Figure 5. Multiplicity dependence of the $\Delta \gamma$ scaled by the geometric mean of the positive and negative particle multiplicities in $\mathrm{d}+\mathrm{Au}$ and $\mathrm{Au}+\mathrm{Au}$ collisions at $\sqrt{s_{N N}}=200 \mathrm{GeV}$ (Particles contributing to the correlators and $v_{2, \text { bbye }}^{\text {obs }}$ are from one half of the TPC in $\eta$, and the event plane is from the other half of the TPC). 


\section{Summary}

Charge dependent three particle correlators are measured in $\mathrm{Au}+\mathrm{Au}$ collisions at $\sqrt{s_{N N}}=200$ $\mathrm{GeV}$. Identified-particle correlations are explored to search for the CME/CVE. The event-by-event anisotropy method and the mixed-event method are exploited to suppress the flow background. In $\mathrm{Au}+\mathrm{Au}$ collisions at $200 \mathrm{GeV}$, with flow background suppression, proton-proton correlation signal becomes negative. In central $\mathrm{Au}+\mathrm{Au}$ collisions form 7.7 to $200 \mathrm{GeV}$, with flow background suppression, charge separation signals are consistent with zero. In non-central collisions, the signals are finite, and the energy dependence is weak. In $\mathrm{d}+\mathrm{Au}$ collisions at $200 \mathrm{GeV}$, we observe sizeable charge dependent signal, comparable to $200 \mathrm{GeV} \mathrm{Au}+\mathrm{Au}$ collisions at similar multiplicities. The analysis of high statistical $\mathrm{p}+\mathrm{Au}, \mathrm{d}+\mathrm{Au}$ data at $200 \mathrm{GeV}$ are under way.

\section{Acknowledgments}

This work was partly supported by the U.S. Department of Energy (Grant No. DE-FG02-88ER40412), and the NSFC of China under Grant Nos. 11505073.

\section{References}

[1] T. D. Lee, Phys. Rev. D8, 1226 (1973).

[2] T. D. Lee and G. C. Wick, Phys. Rev. D9, 2291 (1974).

[3] P. D. Morley and I. A. Schmidt, Z. Phys. C26, 627 (1985).

[4] D. E. Kharzeev, J. Liao, S. A. Voloshin, and G. Wang, Prog. Part. Nucl. Phys. 88, 1 (2016).

[5] K. Fukushima, D. E. Kharzeev, and H. J. Warringa, Phys. Rev. D78, 074033 (2008).

[6] D. Kharzeev, R. D. Pisarski, and M. H. G. Tytgat, Phys. Rev. Lett. 81, 512 (1998); D. Kharzeev and R. D. Pisarski, Phys. Rev. D61, 111901 (2000).

[7] S. A. Voloshin, Phys. Rev. C70, 057901 (2004).

[8] D. Kharzeev, D. T. Son, Phys. Rev. Lett. 106, 062301 (2011).

[9] STAR, B. I. Abelev et al., Phys. Rev. Lett. 103, 251601 (2009).

[10] STAR, B. I. Abelev et al., Phys. Rev. C 81, 054908 (2010).

[11] STAR, L. Adamczyk et al., Phys. Rev. C 88, 064911 (2013).

[12] STAR, L. Adamczyk et al., Phys. Rev. Lett.113, 052302 (2014).

[13] N. N. Ajitanand, S. Esumi, R. A. Lacey (PHENIX Collaboration), in: Proc. of the RBRCWorkshops, vol.96, 230 (2010).

[14] ALICE, B. I. Abelev et al., Phys. Rev. Lett. 110, 012301 (2013).

[15] F. Wang, Phys.Rev. C81, 064902 (2010).

[16] K. H. Ackermann, et al., Nucl. Instr. and Meth. A 499, 624 (2003).

[17] M. Anderson, et al., Nucl. Instr. and Meth. A 499, 659 (2003).

[18] W. J. Llope, et al, doi:10.1016/j.nima.2010.07.086; STAR TOF Proposal (2004).

[19] C. Adler, H. Strobele, A. Denisov, E. Garcia, M. Murray, and S. White, Nucl. Instr. and Meth. A 461, 337 (2001); C. E. Allgower et al. (STAR Collaboration), ibid. 499, 740 (2003);

[20] F. Zhao (for STAR collaboration), Nucl. Phys. A931, 746 (2014).

[21] STAR, L. Adamczyk et al., Phys. Rev. C89, 044908 (2014).

[22] F. Wang and J. Zhao, arXiv:1608.06610[nucl-th].

[23] CMS, V. Khachatryan et al., arXiv:1610.00263 [nucl-ex]. 